\title{
Turbulent Buoyant Jet of a Low-Density Gas Leaks Into a High-Density Ambient: Hydrogen Leakage in Air
}

\author{
M.F. El-Amin and Shuyu Sun \\ King Abdullah University of Science and Technology (KAUST), Thuwal \\ Kingdom of Saudi Arabia
}

\section{Introduction}

The low-density gas jet injected into a high-density ambient has particular interest in several industrial applications such as fuel leaks, engine exhaust, diffusion flames, materials processing, as well as natural phenomena such fires and volcanic eruptions. The most interesting application of this problem nowaday is the hydrogen leaks in air; since when it mixes with air, fire or explosion can result. The expected extensive usage of hydrogen increases the probability of its accidental release from hydrogen vessel infrastructure. Hydrogen energy has much promise as a new clean energy and is expected to replace fossil fuels; however, hydrogen leakage is considered to be an important safety issue and is a serious problem that hydrogen researchers must address. Hydrogen leaks may occur from loose fittings, o-ring seals, pinholes, or vents on hydrogen-containing vehicles, buildings, storage facilities, or other hydrogen-based systems. Hydrogen leakage may be divided into two classes, the first is a rapid leak causing combustion, while the other is an unignited slow-leak. However, hydrogen is ignited in air by some source of ignition such as static electricity (autoignition) or any external source. Classic turbulent jet flame models can be used to model the first class of hydrogen leakage; cf. (Schefer et al., 2006; Houf \& Schefer, 2007; Swain et al., 2007; Takeno et al., 2007). This work is focused on the second class of unignited slow-leaks. Previous work introduced a boundary layer theory approach to model the concentration layer adjacent to a ceiling wall at the impinging and far regions in both planar and axisymmetric cases for small-scale hydrogen leakage El-Amin et al. (2008); El-Amin \& Kanayama (2009a; 2008).

This kind of buoyant jets 'plume' is classed as non-Boussinesq; since the initial fractional density difference is high, which is defined as, $\Delta \rho_{0}=\left(\rho_{\infty}-\rho_{0}\right)$, where $\rho_{0}$ is the initial centerline density (density at the source) and $\rho_{\infty}$ is the ambient density. Generally, for binary selected low-densities gases at temperature $15^{\circ} \mathrm{C}$, the initial fractional density differences are 0.93 for $\mathrm{H}_{2}-\mathrm{Air}, 0.86$ for $\mathrm{He}-\mathrm{Air}, 0.43$ for $\mathrm{CH}_{4}-\mathrm{Air}$ and 0.06 for $\mathrm{C}_{2} \mathrm{H}_{2}-\mathrm{N}_{2}$. (Crapper \& Baines, 1977) suggested that the upper bound of applicability of the Boussinesq approximation is that the initial fractional density difference $\Delta \rho_{0} / \rho_{\infty}$ is 0.05 . In general, one can say that the Boussinesq approximation is valid for small initial fractional density difference, $\Delta \rho_{0} / \rho_{\infty}<<1$. However, in these cases of invalid Boussinesq approximation a density equation must be used. Moreover, a discussion of this classification is given by (Spiegel \& Veronis, 1960); and 
the non-Boussinesq plume was studied, for example, in (Woods, 1997) and (Carlotti \& Hunt, 2005).

The integral method was used by (Agrawal \& Prasad, 2003) to derive similarity solutions for several quantities of interest including the cross-stream velocity, Reynolds stress, the dominant turbulent kinetic energy production term, and eddy diffusivities of momentum and heat for axisymmetric and planar turbulent jets, plumes, and wakes. (El-Amin \& Kanayama, $2009 \mathrm{~b}$ ) used the experimental results by (Schefer et al., 2008b) and others such as the streamwise and centerline velocities, and the concentration at any downstream location with their empirical constants for the momentum-dominated regime of a buoyant jet resulting from hydrogen leakage. In (El-Amin \& Kanayama, 2009c), the similarity form of a non-Boussinesq hydrogen-air buoyant jet resulting from a small-scale hydrogen leakage in the air was developed. In (El-Amin, 2009), non-Boussinesq hydrogen-air buoyant jet resulting from a small-scale hydrogen leakage in the air was investigated. Recently, (El-Amin et al., 2010) introduced analysis of a turbulent buoyant confined jet using realizable $k-\epsilon$ model and several empirical models were developed.

In this chapter, we study the problem of low-density gas jet injected into high-density ambient numerically with an example of hydrogen leakage in air which is important issue faces hydrogen energy developing. The resulting buoyant jet can be divided into two regimes, momentum-dominated and buoyant-dominated. We study each regime indvidualy under certain assumptions and governing laws. Firstly, we used the integral method to derive a complete set of results and expressions for selected physical turbulent properties of the momentum-dominated buoyant jet regime of small-scale hydrogen leakage. In the second part, the buoyancy-dominated regime is investigated.

A jet/plume represents an example of free shear flows, which is produced when the fluid exits a nozzle with initial momentum. In the ideal case of jet/plume, the initial volume flux is assumed to be zero; however, in the real cases, the jet/plume has a finite source size and initial volume flux. In the pure jet "non-buoyant jet" the initial momentum flux as a high velocity injection causes the turbulent mixing. In a pure plume the buoyancy flux causes a local acceleration which lead to turbulent mixing. In the general case of a buoyant jet "forced plume" a combination of initial momentum flux and buoyancy flux is responsible for turbulent mixing. In general, jets are considered the most popular flows on which new theories of turbulence are tested. The popularity can be gauged by the extensive literature that exits about them. Comprehensive review of jets was presented by (Rajaratnam, 1976; List, 1982; Fischer et al., 1979). The behavior of the turbulent vertical round jet and plume were considered by (Turner, 1973; Shabbir \& Georg, 1994). Observations show that, after some distance about $10 d$, where $d$ is the inlet diameter, from the source, the flow becomes turbulent and expands conically; where the conical head (origin) is virtually located close to the source. Upper that $10 \mathrm{~d}$ distance, the flow tends to be self-similar (i.e., the shapes of mean quantities become independent of the axial location when normalized by scales of velocity and length; (Hinze, 1975)). The instantaneous flow field is unsteady, complex and three-dimensional; however, when flow field is averaged over a period that is much larger than the timescale (the ration of the characteristic length scale to the velocity scale) in the flow, the resulting mean flow field is steady and two-dimensional (Papantoniou \& List, 1989; Venkatakrishnan et al., 1999; Bhat \& Narasimha, 1996). The vertical axis passing through the center of the inlet is known as centerline and the mean flow as axisymmetric (symmetric around the centerline). It is worth mentioning that the axisymmetric jets/plumes are the best way for testing both experimental and numerical work. The distance along the centerline is denoted by $\mathrm{z}$ and that 


\begin{tabular}{|l|c|c|c|c|c|c|}
\hline & $b$ & $U_{c l}(z)$ & $C_{c l}(z)$ & $c_{m}$ & $A$ & $\alpha$ \\
\hline Jet & $c_{m}\left(z-z_{0}\right)$ & $\frac{A d U_{0}}{\left(z-z_{0}\right)}$ & $\frac{5.6 \sqrt{\pi} d C_{0}}{2\left(z-z_{0}\right)}$ & $0.1-0.13$ & $5-7$ & $\frac{c_{m}}{2}$ \\
\hline Plume & $c_{m}\left(z-z_{0}\right)$ & $\frac{A F_{0}^{1 / 2}}{\left(z-z_{0}\right)^{1 / 3}}$ & $\frac{9.4 Y}{F_{0}^{1 / 3}\left(z-z_{0}\right)^{5 / 3}}$ & $0.1-0.13$ & $3.9-4.7$ & $\frac{5 c_{m}}{6}$ \\
\hline
\end{tabular}

Table 1. Forms of jet/plume mean flow variables and their related coefficients.

in the horizontal by $r$. Mostly, the mean centerline velocity $U_{\mathcal{c}}$ is taken as the velocity scale, and the jet/plume width, denoted by $b$, is taken as the length scale. The following forms in Table 1 , show the mean flow variables, which are known as similarity solutions and suggested by both experimental observations and dimensional analyses (e.g. (Fischer et al., 1979; Turner, 1973; Hussein et al., 1994; Dai et al., 1995)). Note that, $F_{0}=K d^{2} U_{0} g \Delta \rho_{0} / \rho_{\infty}, K=\pi /\left(1+\lambda^{2}\right)$, $\lambda=c_{m} / c_{\mathcal{c}}$ and, $Y=Q C_{0}=(\pi / 4) d^{2} U_{0} C_{0}, Q=(\pi / 4) d^{2} U_{0}$ In the above equations, $d$ is the nozzle diameter, $c_{m}$ is spread rate of the momentum. $U_{0}$ and $C_{0}$ are, respectively, the vertical velocity and concentration at the inlet. $Q, F_{0}$ and $Y$ are respectively, volume flux, buoyancy flux and mass flux at the inlet. $z_{0}$ is the virtual origin, that is, the distance above/below the orifice where the flow appears to originate. In this study we will assume that both the virtual origin and the jet source have the same location, i.e. $z_{0}=0$, therefore, $z-z_{0}$ in the above expressions will replaced by $z$.

\section{Momentum-dominated regime}

The turbulent round jet is the most suitable model to describe unintended hydrogen releases with circular leak geometries, such as pinholes, through which the flow of hydrogen is an axisymmetric jet. Measurements of the hydrogen distribution in a laboratory-scale hydrogen leak under flow conditions and neglecting the buoyancy effects are described by (Schefer et al., 2008 b). They reported a Froude number of 268, which is in the momentum-dominated regime where the effects of buoyancy-generated momentum are small and the Reynolds number was sufficient for fully developed turbulent flow. Their results showed that hydrogen jets behave similar to jets of helium and conventional hydrocarbon fuels like methane and propane in the momentum-dominated regime. As with any jet flow, the hydrogen mass fraction centerline decay rate shows $z^{-1}$ dependence, where $z$ is the axial distance from the jet exit. In this section, we used the integral method to derive a complete set of results and expressions for selected physical turbulent properties of momentum-dominated buoyant jet regime of small-scale hydrogen leakage El-Amin \& Kanayama (2009b). Several quantities of interest, including the cross-stream velocity, Reynolds stress, velocity-concentration correlation (radial flux), dominant turbulent kinetic energy production term, turbulent eddy viscosity and turbulent eddy diffusivity, are obtained. In addition, the turbulent Schmidt number is estimated and the normalized jet-feed material density and the normalized momentum flux density are correlated. Experimental results from (?) and other works for the momentum-dominated jet resulting from small-scale hydrogen leakage are used in the integral method. For a non-buoyant jet or momentum-dominated regime of a buoyant jet, both the centerline velocity and centerline concentration are proportional with $z^{-1}$. The effects of buoyancy-generated momentum are assumed to be small, and the Reynolds number is sufficient for fully developed turbulent flow. The hydrogen-air momentum-dominated regime or non-buoyant jet is compared with the air-air jet as an example of non-buoyant jets. Good agreement was found between the current results and experimental results from the literature. In addition, the turbulent Schmidt number was shown to depend solely on the ratio of the momentum 


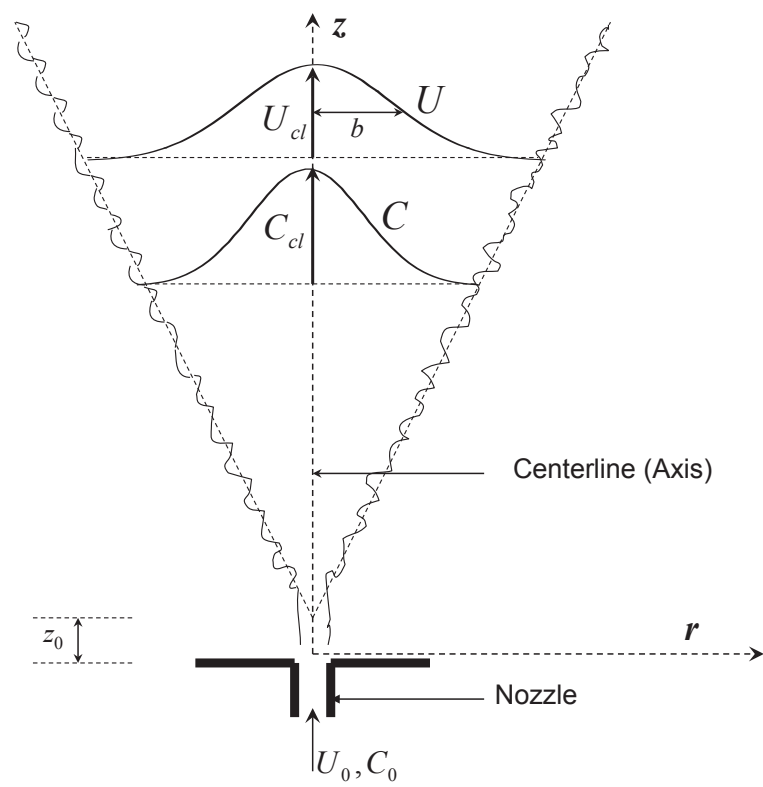

Fig. 1. Schematic of turbulent jet geometry.

spread rate to the material spread rate. Standard empirical Gaussian expressions for the mean streamwise velocity $U$ and concentration $C$ are substituted into the governing equations of the momentum-dominated regime of a buoyant hydrogen jet, in order to derive a number of turbulent quantities using integral methods. In the first step, the expression for $U$ is substituted into the continuity equation and a centerline velocity variation is assumed, and the equation is integrated to determine the mean cross-stream velocity profile $V$. In the second step, the expressions for $U$ and $V$ are substituted into the simplified momentum equation and integrated to determine the Reynolds stress. In the third step, the expressions for $U$ and $V$ are substituted into the simplified concentration equation and integrated to determine the velocity-concentration correlation.

\subsection{Governing equations}

Viscous stresses are assumed to be much smaller than the corresponding turbulent shear stresses provided that the nozzle Reynolds number is about a few thousand (Rajaratnam, 1976). In self-similar region, the axisymmetric streamwise governing equations in cylindrical coordinates (see Fig.1) obtained using simplifications of (Refs. (Agrawal \& Prasad, 2003; Bhat \& Narasimha, 1996; Chen \& Rodi, 1980; Tennekes \& Lumley, 1972; Gebhart et al., 1988; Rajaratnam, 1976)) the continuity, momentum, and concentration equations in cylindrical coordinates for a vertical axisymmetric momentum-dominated regime of buoyant hydrogen free jet can be written as,

$$
\frac{\partial(r V)}{\partial r}+\frac{\partial U}{\partial z}=0
$$




$$
\begin{aligned}
& V \frac{\partial U}{\partial r}+U \frac{\partial U}{\partial z}+\frac{1}{r} \frac{\partial(r \overline{u v})}{\partial r}=0 \\
& V \frac{\partial C}{\partial r}+U \frac{\partial C}{\partial z}+\frac{1}{r} \frac{\partial(r \overline{v c})}{\partial r}=0
\end{aligned}
$$

The overbar denotes that the time-averaged quantities $u$ and $v$ are the components of velocity fluctuations on the axisymmetric coordinates $r$ and $z$, respectively. $c$ is the concentration fluctuation, $v$ is the kinematic viscosity, and $D$ is the mass molecular diffusivity of hydrogen. The streamwise velocity and concentration at any downstream location for a self-similar axisymmetric jet, can be approximated by a Gaussian distribution (Agrawal \& Prasad, 2003; Bhat \& Narasimha, 1996; Turner, 1986; List, 1982; Chen \& Rodi, 1980; Agrawal \& Prasad, 2002),

$$
\begin{gathered}
U(r, z)=U_{c l}(z) \exp \left(-\frac{r^{2}}{b^{2}(z)}\right) \\
C(r, z)=C_{c l}(z) \exp \left(-\lambda^{2} \frac{r^{2}}{b^{2}(z)}\right)
\end{gathered}
$$

The centerline velocity $U_{c l}$ and the centerline concentration $C_{c l}$ vary with $z^{-1}$, while the jet width $b$ increases linearly with $z$ (Agrawal \& Prasad, 2003; Tennekes \& Lumley, 1972; Bhat \& Krothapalli, 2000). Dimensional arguments together with experimental observations suggest that the mean flow variables, which are known as similarity solutions, are as follows ((Fischer et al., 1979; Hussein et al., 1994; Shabbir \& Georg, 1994; Schefer et al., 2008b)),

$$
\begin{gathered}
U_{c l}(z)=\frac{A d U_{0}}{z-z_{0}} \\
C_{c l}(z)=\frac{d^{*}}{K_{C}\left(z-z_{0}\right)}=\frac{d\left(\rho_{H_{2}} / \rho_{\text {air }}\right)^{1 / 2}}{K_{C}\left(z-z_{0}\right)} \\
b(z)=c_{m}\left(z-z_{0}\right)
\end{gathered}
$$

In the above equations, $d$ and $U_{0}$ are the nozzle diameter and the vertical velocity at the inlet, respectively (Fig.1). $z_{0}$ is the virtual origin, which is the distance above/below the orifice where the flow appears to originate. The experimentally measured spread rate $c_{m}$ varies in the range 0.1-0.13 and the constant $A$ varies between 5-7 Fischer et al. (1979); Hussein et al. (1994). For this investigation, the value of the spread rate for the hydrogen jet of $c_{m}=0.103$ was used, where this was experimentally determined by Schefer et al. [1]. The spread rate for the concentration $c_{c}$ is given in the formula $C=C_{c l} \exp \left(-r^{2} /\left[c_{c}^{2}\left(z-z_{0}\right)\right]\right)$. It is well known that $c_{\mathcal{c}} \neq c_{m}, \lambda=c_{m} / c_{\mathcal{c}}$, meaning that the velocity and concentration spread have different rates. The corresponding streamwise concentration for the hydrogen free jet given by Schefer et al. (2008b) is $C=C_{c l} \exp \left(-59 r^{2} /\left(z-z_{0}\right)\right)$, which gives $59=1 / c_{c}^{2}$ and $c_{c}=0.13, \lambda=0.79$. The expression for the streamwise concentration may then be rewritten as:

$$
C(r, z)=C_{c l}(z) \exp \left(-\lambda^{2} \eta^{2}\right)=C_{c l}(z) \exp \left(-0.63 \eta^{2}\right)
$$

where $\eta=r / b$. 


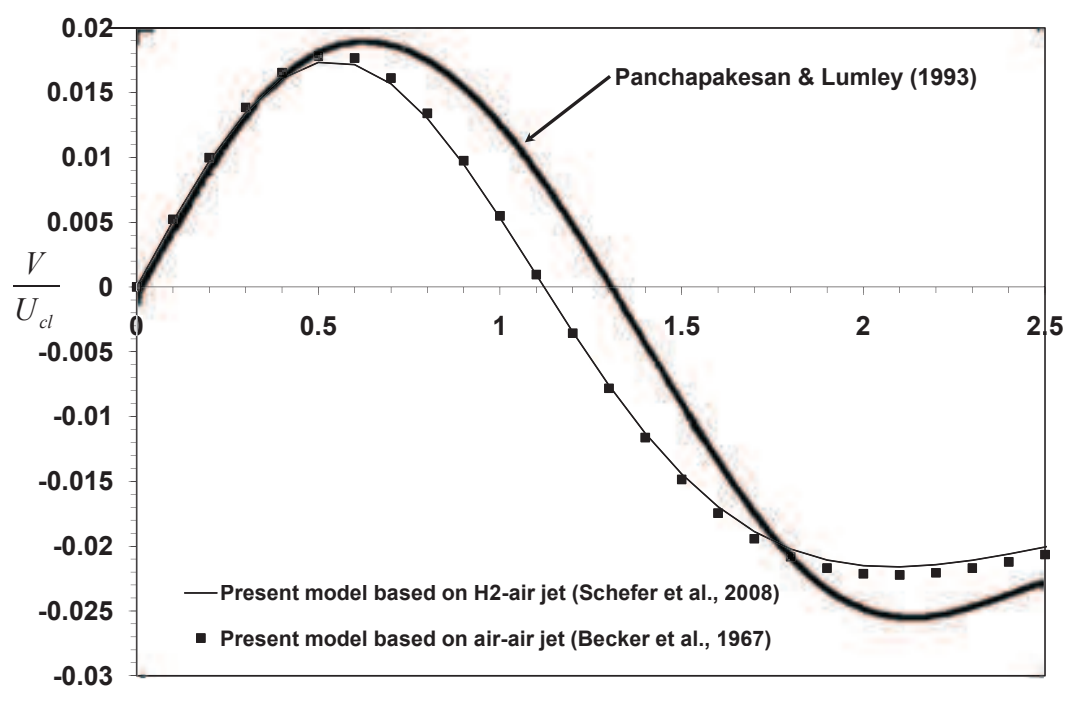

n

Fig. 2. Cross-stream velocity profile for the momentum-dominated jet.

\subsection{Mean and turbulent quantities}

The continuity equation for the time-averaged velocities can be solved by substituting from (4) and (6) into (1) to obtain the cross-stream velocity in the form:

$$
\frac{V}{U_{c l}}=\frac{c_{m}}{2 \eta}\left(-1+\exp \left(-\eta^{2}\right)+2 \eta^{2} \exp \left(-\eta^{2}\right)\right)
$$

Fig.2 shows the normalized cross-stream velocity profiles $V / U_{c l}$ plotted against the normalized coordinate $\eta$. Since the case under study is a momentum-dominated (non-buoyant) jet, it is comparable with the air-air jet. The normalized cross-stream velocity profiles $V / U_{c l}$ (Eq. (12)) are based on measurements of a hydrogen-air jet by (Schefer et al., 2008b) and an air-air jet by (Becker et al., 1967), which are compared with measurements of an air-air jet by (Panchapakesan \& Lumley, 1993), as illustrated in Fig. 2. From this figure, it can be seen that the cross-stream velocity vanishes at the centerline and becomes outward in the neighborhood of the centerline $(0 \leq \eta \leq 1.12$ for a hydrogen-air jet and an air-air jet according to Eq. (12), and $0 \leq \eta \leq 1.31$ for measurements of an air-air jet from (Panchapakesan \& Lumley, 1993)). A maximum is then reached (0.0173 for a hydrogen-air jet and 0.0178 for an air-air jet according to Eq. (12) at $\eta=0.5$, and 0.0188 at $\eta=0.62$ for measurements of an air-air jet (Panchapakesan \& Lumley, 1993)), and then declines back to zero $(\eta=1.12$ for a hydrogen-air jet and an air-air jet according to Eq. (10), and $\eta=1.31$ for measurements of an air-air jet Panchapakesan \& Lumley (1993)). In addition, the cross-stream profile's flow becomes inward $\left(V / U_{c l}<0\right)$, reaches a minimum $(-0.0216$ for a hydrogen-air jet and -0.0222 for an air-air jet at $\eta=2.1$ according to Eq. (10), and -0.026 at $\eta=2.15$ for measurements of an air-air jet Panchapakesan \& Lumley (1993)) and reaches an asymptote of 0 as $\eta \rightarrow \infty$. The differences between maximum and minimum values of the cross-stream velocities noted above are attributed to the high Reynolds number $(R e=11000)$ used in Ref. (Panchapakesan \& Lumley, 1993). In addition, it is noteworthy that the cross-stream velocity asymptotes to zero $(V \rightarrow 0)$ much more slowly than the streamwise velocity $U$ does. Thus, although the central 


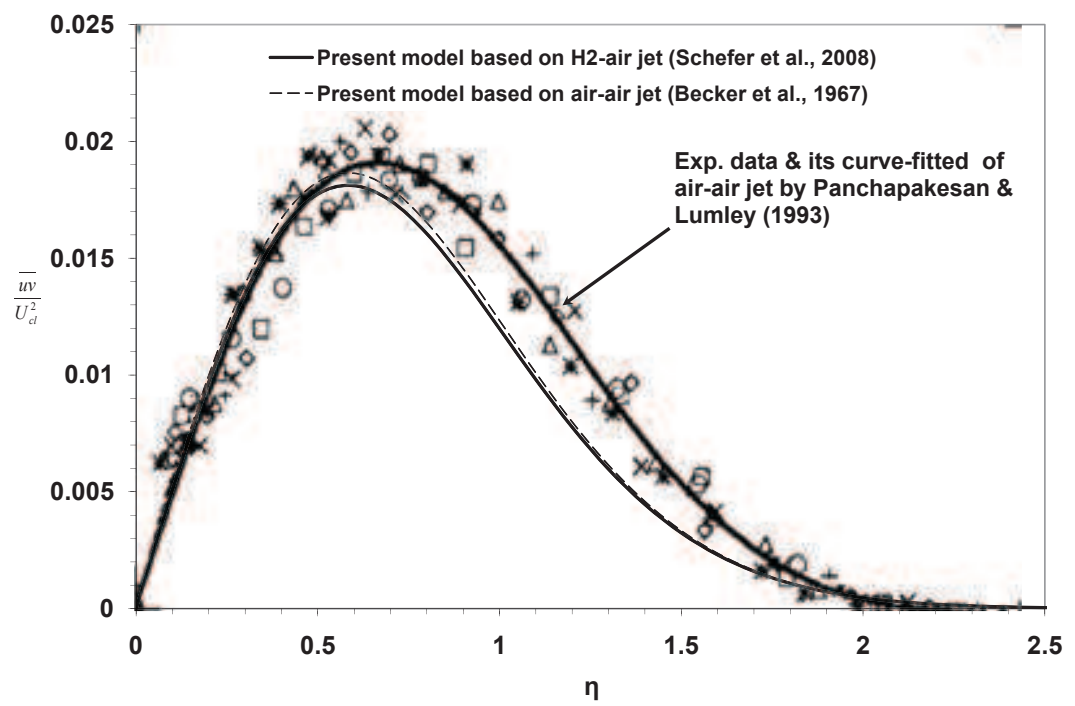

Fig. 3. Reynolds stress profile for the momentum-dominated jet.

region is dominated by the axial component of velocity, the cross-stream flow predominates far away from it (Agrawal \& Prasad, 2003). From Eq. (10), $\lim _{\eta \rightarrow \infty}\left(V / U_{c l}\right)=-c_{m} / 2 \eta$. The inward extension of the curve $c_{m} / 2 \eta$ intersects the edge of the jet $(\eta=1)$ with a value of $c_{m} / 2$ ( 0.0515 for the hydrogen-air jet and 0.053 for the air-air jet). The incremental volume flux can be used to define the entrainment coefficient $\alpha$ (see Turner (1986)):

$$
\frac{d \mu}{d z}=2 \pi b U_{c l} \alpha
$$

where / $m u$ is the volume flux for axisymmetric jet given by:

$$
\mu=\int_{0}^{\infty} 2 \pi r U(r) d r
$$

From (Agrawal \& Prasad, 2003), $d \mu / d z$ is the incremental volume flux entering the jet through a circular control surface at large $r$,

$$
\frac{d \mu}{d z}=\lim _{\eta \rightarrow \infty}(-2 \pi r V)=\pi b c_{m} U_{c l}
$$

Eqs. (11) and (13) give the entrainment coefficient $\alpha=c_{m} / 2=0.0515$ for the momentum-dominated hydrogen-air jet and $\alpha=c_{m} / 2=0.053$ for the air-air jet based on measurements by (Becker et al., 1967).

By inserting the time-averaged profiles of $U$ and $V$, Eq. (4) and Eq. (10), respectively, into the momentum equation, Eq. (4), we obtain the time-averaged profile for the Reynolds stress $\overline{u v}$ in the form:

$$
\frac{\overline{u v}}{U_{c l}^{2}}=\frac{c_{m}}{2 \eta} \exp \left(-\eta^{2}\right)\left(1-\exp \left(-\eta^{2}\right)\right)
$$


The normalized Reynolds stress $\overline{u v} / U_{c l}^{2}$ (Eq. (14)), based on measurements of a hydrogen-air jet by (Schefer et al., 2008b) and an air-air jet by (Becker et al., 1967), are compared with measurements of an air-air jet by (Panchapakesan \& Lumley, 1993), as shown in Fig. 3. This figure shows that the maximum Reynolds stress $\overline{u v} / U_{c l}^{2}$ has a value of $(0.0181$ for a hydrogen-air jet and 0.0186 for an air-air jet according to Eq. (14) and at $\eta=0.6$, and 0.019 at $\eta \approx 0.68$ for measurements of an air-air jet (Panchapakesan \& Lumley, 1993)). It is notable that the fitted-curve for the measurements of (Panchapakesan \& Lumley, 1993) is used for this comparison.

In the same manner used above, the time-averaged profiles of $U$ and $C$ are inserted into Eq. (3), and the time-averaged profile for the velocity-concentration correlation (radial flux) $\overline{v c}$ takes the form:

$$
\frac{\overline{v c}}{U_{c l}^{2}}=\frac{c_{m}}{2 \eta} \exp \left(-\lambda \eta^{2}\right)\left(1-\exp \left(-\eta^{2}\right)\right)
$$

The normalized velocity-concentration correlation (radial flux) $\overline{v c} / U_{c l}^{2}$ (Eq. (15)) based on measurements of hydrogen-air jet by (Schefer et al., 2008b) and air-air jet by (Becker et al., 1967) are compared with measurements of a helium-air jet by (Panchapakesan \& Lu, 1993), as shown in Fig. 4. This figure shows that the maximum radial flux has a value of 0.021 for the hydrogen-air jet and 0.023 for the air-air jet, according to Eq. (14) at $\eta=0.7$, and 0.02 at $\eta \approx 0.88$ for measurements (fitted-curve) of a helium-air jet (Panchapakesan \& Lu, 1993).

The dominant kinetic energy production term is defined by $\overline{u v} \partial U / \partial \eta$. Using Eqs. (4), (6) and (14), gives:

$$
\frac{\overline{u v}}{U_{c l}^{3}} \frac{\partial U}{\partial \eta}=c_{m} \exp \left(-2 \eta^{2}\right)\left(1-\exp \left(-\eta^{2}\right)\right)
$$

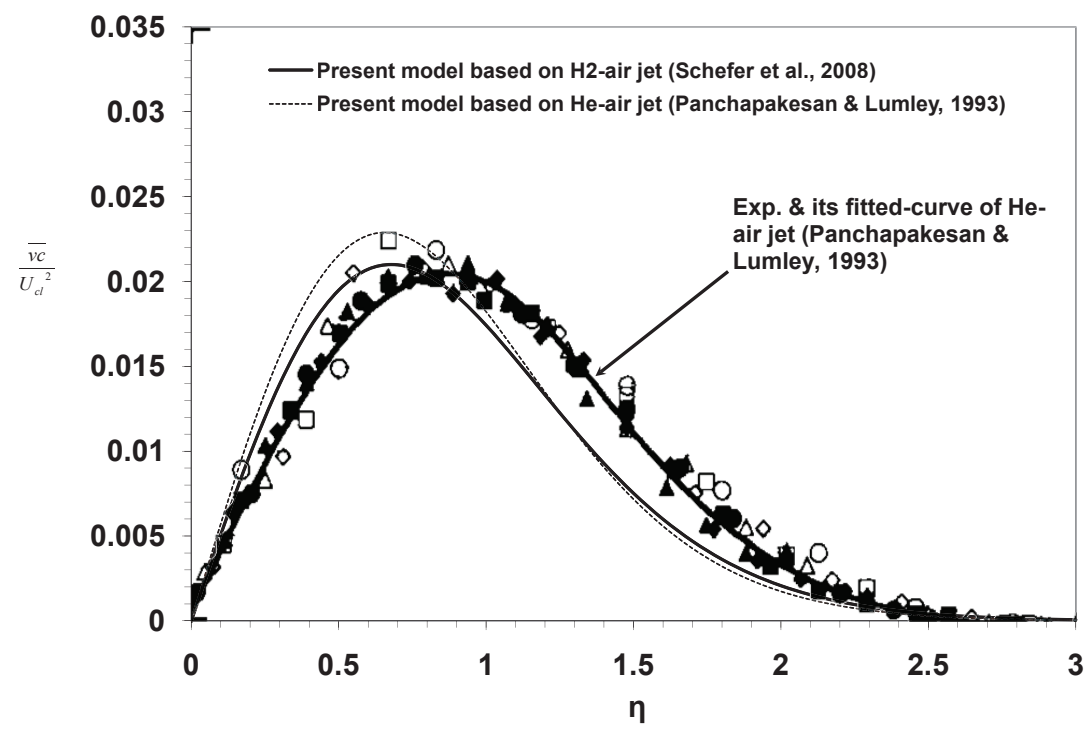

Fig. 4. Velocity-concentration correlation profile for the momentum-dominated jet. 


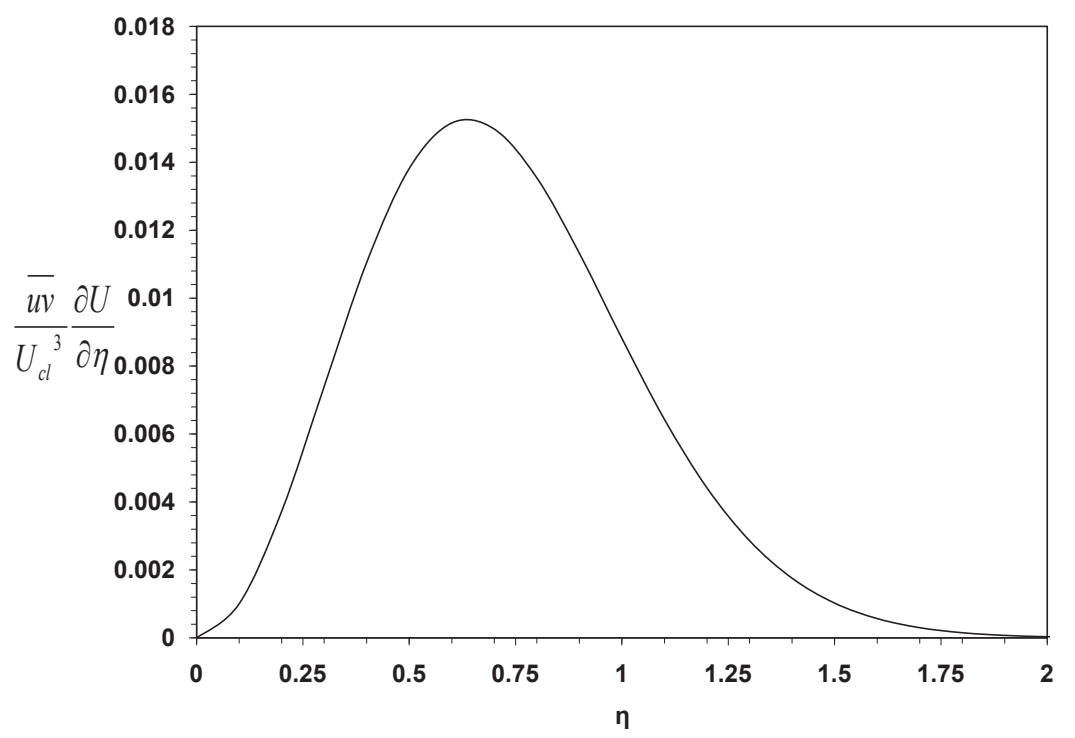

Fig. 5. Dominant kinetic energy production term profile for the momentum-dominated jet.

The complete turbulent kinetic energy equation can be found in the literature (e.g., see Wygnanski). Fig. 5 indicates that the dominant kinetic energy production term reduces to zero for large $\eta$, and has a maximum value of 0.015 at $\eta=0.6$ for the hydrogen-air jet based on the measurements by (Schefer et al., 2008b). The following definitions for the turbulent eddy viscosity $v_{t}$ and turbulent eddy diffusivity $D_{t}$ may also be used,

$$
\overline{u v}=-v_{t} \frac{\partial U}{\partial r}
$$

and

$$
\overline{v c}=-D_{t} \frac{\partial C}{\partial r}
$$

to derive corresponding expressions as,

$$
\frac{v_{t}}{U_{c l} b}=\frac{c_{m}}{4 \eta^{2}}\left(1-\exp \left(-\eta^{2}\right)\right)
$$

and

$$
\frac{D_{t}}{U_{c l} b}=\frac{c_{m}}{4 \lambda^{2} \eta^{2}}\left(1-\exp \left(-\eta^{2}\right)\right)
$$

respectively. Eqs. (19) and (20) indicate that the turbulent eddy viscosity and turbulent eddy diffusivity are not independent of $\eta$, as assumed in works such as (Tennekes \& Lumley, 1972), but are variable as a function of $\eta$, as suggested by others (Lessen, 1978). Both the normalized turbulent eddy viscosity $v_{t} /\left(U_{c l} b\right)$, Eq. (19), and the normalized turbulent eddy diffusivity $D_{t} /\left(U_{c l} b\right)$, Eq. (20) for the hydrogen-air jet based on the measurements by (Schefer et al., 2008b) are plotted in Fig.15. This figure shows that both $v_{t} /\left(U_{c l} b\right)$ and $D_{t} /\left(U_{c l} b\right)$ have 
maximum values of 0.026 and 0.041 , respectively, at the centerline $(\eta=0)$ of the jet and decay to 0 for large $\eta$. It is also interesting that the turbulent eddy diffusivity is greater than turbulent eddy viscosity. If the effects of molecular transport are neglected, the turbulent Schmidt number in the free turbulent flow may be defined as the quantitative difference between the turbulent transport rate of material and momentum. In other words, the turbulent Schmidt number, $S c_{t}=v_{t} / D_{t}$, is a ratio of the eddy viscosity of the turbulent flow $v_{t}$ to the eddy material diffusivity $D_{t}$. It is very well known from literature that the spreading rate of material in free jets is greater than that of momentum; thus, the turbulent Schmidt number is less than unity and has a significant effect on the predictions of species spreading rate in jet flows.

The turbulent eddy viscosity $v_{t}$ and the turbulent eddy diffusivity $D_{t}$, given by Eqs. (19) and (20), respectively, give the turbulent Schmidt number as:

$$
S c_{t}=\frac{v_{t}}{D_{t}}=\lambda^{2}
$$

It is interesting to note that the turbulent Schmidt number depends only on the coefficient $\lambda$, which, in turn, depends on the material. Using Eq. (21), the turbulent Schmidt number $S c_{t}$ for hydrogen momentum-dominated jet is 0.63 , based on the experimental results given by (Schefer et al., 2008b). However, the same formula (Eq. (21)) used with experimental results of the helium-air jet, $c_{m}=0.116, c_{c}=0.138$ (Panchapakesan \& Lu, 1993), the turbulent Schmidt number $S c_{t}$ becomes 0.7. Irrespective of the turbulence structure, the constant value of the turbulent Schmidt number across the whole flow field implies that the momentum process is similar to the material-transport process (He et al., 1999; Yimer et al., 2002).

According to Eq. (21), if $\lambda^{2}$ is replaced by $S c_{t}$ in Eq. (9), the streamwise concentration for the momentum-dominated jet may be rewritten as:

Eqs. (4) and (5) give,

$$
C(r, z)=C_{c l}(z) \exp \left(-S c_{t} \eta^{2}\right)
$$

$$
\frac{U}{U_{c l}}=\exp \left(-\eta^{2}\right)
$$

Therefore, Eq. (22) becomes:

$$
\frac{C}{C_{c l}}=\left(\frac{U}{U_{c l}}\right)^{S c_{t}}
$$

The normalized streamwise momentum flux density across an isothermal jet is given by:

$$
G=\left(\frac{U}{U_{c l}}\right)^{2}
$$

and the normalized jet-feed material density is given by:

$$
W=\frac{U C}{U_{c l} C_{c l}}
$$

Therefore, the mean profiles of jet-feed concentration and streamwise velocity are related by:

$$
W=G^{\left(1+S c_{t}\right) / 2}
$$

Fig. 6 shows a plot of the correlation of the normalized jet-feed material density against the normalized momentum flux density for the hydrogen-air jet, $S c_{t}=0.63$, helium-air jet, $S c_{t}=$ 0.7 , and air-air jet, $S c_{t}=0.76$. 


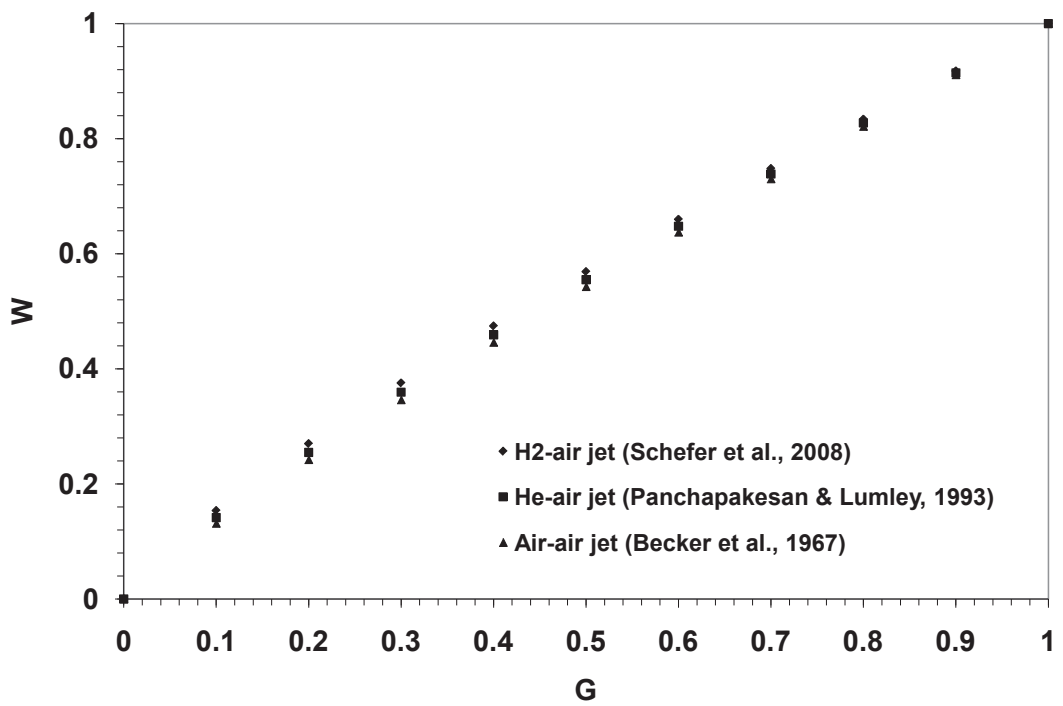

Fig. 6. Correlation of the normalized jet-feed material density against the normalized momentum flux density for hydrogen, helium and air jets.

\section{Buoyancy-dominated regime}

In this part, we study the buoyancy-dominated regime. It is assumed that the local rate of entrainment is consisted from two components; one is the component of entrainment due to jet momentum while the other is the component of entrainment due to buoyancy. The integral models of the mass, momentum and concentration fluxes are obtained and transformed to a set of ordinary differential equations using some similarity transformations El-Amin (2009); El-Amin \& Kanayama (2009c). The resulting system is solved to determine the centerline quantities which are used to get the mean axial velocity, mean concentration and mean density of the jet. Therefore, the centerline and mean quantities are used together with the governing equation to determine some important turbulent quantities such as, cross-stream velocity, Reynolds stress, velocity-concentration correlation, turbulent eddy viscosity and turbulent eddy diffusivity.

\subsection{Governing equations}

Consider a vertical axisymmetric non-Boussinesq buoyant jet resulting from injecting a low-density gas jet into high-density ambient. Using cylindrical polar coordinates $(z, r)$ with the $z$-axis vertical and the source at $z=0$ (see Fig. 1, the continuity, momentum and concentration equations of the steady vertical axisymmetric buoyant free jet can be written as (Agrawal \& Prasad, 2003; Bhat \& Narasimha, 1996; Chen \& Rodi, 1980; Gebhart et al., 1988; Rajaratnam, 1976; Tennekes \& Lumley, 1972; El-Amin, 2009),

$$
\begin{gathered}
\frac{\partial(r \rho V)}{\partial r}+\frac{\partial(r \rho U)}{\partial z}=0 \\
\frac{\partial(r \rho U V)}{\partial r}+\frac{\partial\left(r \rho U^{2}\right)}{\partial z}+\frac{\partial(r \rho \overline{u v})}{\partial r}=g r\left(\rho-\rho_{\infty}\right)
\end{gathered}
$$




$$
\frac{\partial(r \rho V C)}{\partial r}+\frac{\partial(r \rho U C)}{\partial z}+\frac{\partial(r \rho \overline{v C})}{\partial r}=0
$$

where $U$ is the mean streamwise velocity, $V$ is the mean cross-stream velocity profile, $C$ is the hydrogen concentration (mass fraction). The overbar denotes the time-averaged quantities, $u, v$ are the components of velocity fluctuations on the axisymmetric coordinates $r, z$, respectively. $c$ is the concentration fluctuation, $\rho$ is the mixture density and $D$ is the mass molecular diffusivity of the hydrogen in air. On the other hand, from the experimental observations, the equations for the vertical velocity, density deficiency and mass fraction profiles with assuming that the hydrogen-air mixture behaves as an ideal gas, are as follows,

$$
\begin{gathered}
U(r, z)=U_{c l}(z) \exp \left(-\frac{r^{2}}{b^{2}(z)}\right) \\
\rho_{\infty}-\rho(r, z)=\left(\rho_{\infty}-\rho_{c l}(z)\right) \exp \left(-\lambda^{2} \frac{r^{2}}{b^{2}(z)}\right) \\
\rho(r, z) C(r, z)=\rho_{c l}(z) C_{c l}(z) \exp \left(-\lambda^{2} \frac{r^{2}}{b^{2}(z)}\right)
\end{gathered}
$$

where $U(r, z)$ and $\rho(r, z)$ are the mean velocity and mean density at any point of the jet body, $U_{c l}(z)$ and $\rho_{c l}(z)$ are the centerline velocity and density. The buoyancy spreading factor $\lambda=$ $c_{m} / c_{c}$ expresses the difference in spreading rates between the velocity and density deficiency profiles. It is well known that $c_{m} \neq c_{\mathcal{C}}$, i.e., velocity and density spread in different rates. The mass fraction concentration $C$ is related to the mixture density by,

$$
\rho=\frac{1}{\left.\left[\left(1 / \rho_{0}\right)-1 / \rho_{\infty}\right)\right] C+\left(1 / \rho_{\infty}\right)}
$$

while, the mole fraction concentration $X$ is related to the mixture density by,

$$
\rho=\rho_{\infty}(1-X)+\rho_{0} X
$$

Therefore, the mass fraction and mole fraction can be related as follows,

$$
C=\frac{\rho_{0} X}{\rho_{\infty}(1-X)+\rho_{0} X}=\frac{R_{\rho} X}{1+\left(R_{\rho}-1\right) X}
$$

where $R_{\rho}=\frac{\rho_{0}}{\rho_{\infty}}$ is the density ratio. Also, one can note that $\rho C=\rho_{0} X$, and therefore the equation of concentration, Eq. (30), can be rewritten in terms of mole fraction $X$ as follows,

$$
\frac{\partial(r \rho V X)}{\partial r}+\frac{\partial(r \rho U X)}{\partial z}+\frac{1}{\rho_{0}} \frac{\partial(r \rho \overline{v c})}{\partial r}=0
$$

or,

$$
\frac{\partial(r \rho V X)}{\partial r}+\frac{\partial(r \rho U X)}{\partial z}+\frac{\partial(r \rho \overline{v x})}{\partial r}=0
$$

where $x$ is the corresponding turbulent fluctuation of the mole fraction concentration. 


\subsection{Entrainment coefficient}

The turbulent entrainment of the turbulent buoyant jet is defined as; the ambient fluid is mixed across the jet edge and becomes incorporated into the body of the jet. This process has the effect of increasing the jet volume flux and increasing/decreasing the jet density. The turbulent entrainment is usually parameterized by relating the inflow velocity to the mean flow in the jet body; instead of using details depend on transfers of mass and momentum at small scales which are very difficult to compute. (Houf \& Schefer, 2008) have reported from that the local rate of entrainment increases as the jet leaves the momentum-dominated region and enters a region where the effects of buoyancy become more pronounced. The entrainment coefficient is given by,

$$
\alpha=\frac{E}{2 \pi U_{c l} b}
$$

According to this expression, the value of $\alpha$ increases gradually as the height increases from jet region to plume region passing through the intermediate region. However, it has been experimentally observed that the value of the entrainment constant $\alpha$ approaches the limiting value of $\alpha=0.82$ for pure plume. Therefore the expression, Eq. (39), is used until reach the value 0.082 then it will be enforced to be equal to this value from this point and after. The local rate of entrainment may be given in the form,

$$
\begin{gathered}
E=E_{\text {mom }}+E_{\text {buoy }} \\
E_{\text {mom }}=0.282\left(\frac{\pi d^{2}}{4} \frac{\rho_{0} U_{0}^{2}}{\rho_{\infty}}\right)^{\frac{1}{2}}
\end{gathered}
$$

Here, $E_{\text {mom }}$ is the component of entrainment due to jet momentum (Houf \& Schefer, 2008; Ricou \& Spalding, 1961), while, $E_{\text {buoy }}$ is the component of entrainment due to buoyancy (Hirst, 1971).

$$
E_{\text {buoy }}=\frac{2 \pi U_{c l} b a_{2}}{F r_{1}}
$$

where $F r_{1}$ is the local Froude number which is defined as,

$$
F r_{1}=\frac{U_{c l}^{2} \rho_{0}}{g d\left(\rho_{\infty}-\rho_{c l}\right)}
$$

The local Froude number $F r_{1}$ is high in the momentum-dominate region therefore $E_{b u o y}$ term can be neglected compared to $E_{\text {mom }}$ in the total entrainment expression, Eq. (40). However, as the buoyancy effect become more important, the local Froude number $F r_{1}$ decreases and $E_{\text {buoy }}$ term begins to contribute. Determination of the constant $a_{2}$ was developed by (Houf \& Schefer, 2008), based on the experiment of the vertical hydrogen jet, to be dependent on the densimetric Froude number Fr.

$$
a_{2}= \begin{cases}17.313-0.11665 F r+2.0771 \times 10^{-4} F r & \forall F r<268 \\ 0.97 & \forall F r \geq 268\end{cases}
$$

The densimetric Froude number $F r$ is given by, 


$$
F r=\left(\frac{U_{0}^{2} \rho_{0}}{g d\left(\rho_{\infty}-\rho_{0}\right)}\right)^{\frac{1}{2}}
$$

\subsection{Similarity solutions}

Integrating the continuity equation (28) radially, gives,

$$
\frac{d}{d z} \int_{0}^{\infty} r U(r, z) \rho(r, z) d r=-[r \rho V(r, z)]_{r=\infty}=-\rho_{\infty}[r V(r, z)]_{r=\infty}
$$

Since $U(r, z)$ is negligible for $r>b$, then integrating Eq. (28) for $b<r<\infty$ gives,

$$
\int_{b}^{\infty} \frac{\partial}{\partial r}(r V(r, z) \rho(r, z) d r=0
$$

Therefore, this implies to,

$$
-[r V(r, z)]_{r=\infty}=b V_{e}
$$

where $V_{e}$ denotes the inflow velocity at the plume edge which is known as entrainment velocity. Thus we have,

$$
\frac{d}{d z} \int_{0}^{\infty} r U(r, z) \rho(r, z) d r=b V_{e} \rho_{\infty}
$$

This equation indicates that the increase in plume volume flux is supplied by a radial influx from the far field which in turn implies a flow across the plume boundary $b$. (Batchelor, 1954) concluded that a vigorous entrainment of the ambient will be gotten as the density ratio tends to unity, $\rho_{c l} / \rho_{\infty} \rightarrow 1$, while as the density ratio tends to zero, $\rho_{c l} / \rho_{\infty} \rightarrow 0$, the entrainment falls to zero and as the density ratio varies between the two limits there will be a smooth transition. The experiments by (Ricou \& Spalding, 1961) suggests that the entrainment assumption for the entrainment coefficient $\alpha$, and arbitrary density ratio should be given in the form,

$$
V_{e}=\alpha\left(\frac{\rho_{c l}}{\rho_{\infty}}\right)^{\frac{1}{2}} U_{c l}, \quad V_{0}\left(\rho_{c l} / \rho_{\infty} \rightarrow 1\right)=\alpha U_{c l}, \quad V_{0}\left(\rho_{c l} / \rho_{\infty} \rightarrow 0\right)=0
$$

Also, (Morton, 1965) assumed that the rate of entrainment into a strongly buoyant plume is a function of both density ratio $\rho_{c l} / \rho_{\infty}$ and depends on Reynolds stresses which have local magnitude proportional to $\rho_{c l} U_{c l}^{2}$ and hence on dimensional grounds, it seems reasonable to assume a local entrainment velocity of $\alpha\left(\rho_{c l} / \rho_{\infty}\right)^{1 / 2} U_{c l}$. A similar assumption has been made by (Thomas, 1963) and (Steward, 1970); and results were suggested theoretically by (Townsend, 1966). Therefore, the Eq. (49) can be written in the form,

$$
\frac{d}{d z} \int_{0}^{\infty} r U(r, z) \rho(r, z) d r=b \rho_{\infty} \alpha\left(\frac{\rho_{c l}(z)}{\rho_{\infty}}\right)^{1 / 2} U_{c l}(z)
$$

Finally, the mass flux equation becomes,

$$
\frac{d}{d z} \int_{0}^{\infty} 2 \pi r U(r, z) \rho(r, z) d r=2 \pi b \rho_{\infty} \alpha\left(\frac{\rho_{c l}(z)}{\rho_{\infty}}\right)^{1 / 2} U_{c l}(z)
$$

Now, let us integrate Eq. (29) with respect to $r$ from $r=0$ to $r=\infty$, with noting that $|r \rho U V|_{0}^{\infty}=$ 0 and $|r \rho \overline{u v}|_{0}^{\infty}=0$, we get, 


$$
\frac{d}{d z} \int_{0}^{\infty} r U^{2}(r, z) \rho(r, z) d r=\int_{0}^{\infty} r g\left(\rho_{\infty}-\rho(r, z)\right) U_{c l}(r, z) d r
$$

or,

$$
\frac{d}{d z} \int_{0}^{\infty} 2 \pi r U^{2}(r, z) \rho(r, z) d r=\int_{0}^{\infty} 2 \pi r g\left(\rho_{\infty}-\rho(r, z)\right) U_{c l}(r, z) d r
$$

Similarly, integrating Eq. (30) with respect to $r$ from $r=0$ to $r=\infty$, with noting that $|r \rho C V|_{0}^{\infty}=$ 0 and $|r \rho \overline{u c}|_{0}^{\infty}=0$, we get,

$$
\frac{d}{d z} \int_{0}^{\infty} r U(r, z) \rho(r, z) C(r, z) d r=0
$$

or,

$$
\frac{d}{d z} \int_{0}^{\infty} 2 \pi r U(r, z) \rho(r, z) C(r, z) d r=0
$$

This equation may be equivalent to the buoyancy flux equation which can be given the following form (Crapper \& Baines, 1977),

$$
\frac{d}{d z} \int_{0}^{\infty} 2 \pi r U(r, z)\left(\rho_{\infty}-\rho(r, z)\right) d r=0
$$

Substituting Eqs. (31)- (33) into Eq. (57) it reduces to,

$$
\frac{d}{d z}\left(b^{2} U_{c l}(z)\left(1-\frac{\rho_{c l}(z)}{\rho_{\infty}}\right)\right)=0
$$

Now, let us define the buoyancy flux $F$ as,

$$
F=K b^{2}(z) U_{c l}(z) g\left(1-\frac{\rho_{c l}(z)}{\rho_{\infty}}\right)
$$

where $K=\pi /\left(1+\lambda^{2}\right)$

Since the buoyancy flux is conserved, we have

$$
F=F_{0}=K d^{2} U_{0} g\left(1-\frac{\rho_{0}}{\rho_{\infty}}\right)=K d^{2} U_{0} g \frac{\Delta \rho_{0}}{\rho_{\infty}}
$$

Substituting Eqs. (32), (33), (58) and (60) into Eqs. (52), (54) and (56) gives,

$$
\begin{aligned}
\frac{d}{d z}\left(b^{2}(z) U_{c l}(z)\right) & =2 \alpha b U_{c l}\left(1-\frac{F_{0}}{K g b^{2}(z) U_{c l}(z)}\right)^{1 / 2} \\
\frac{d}{d z}\left(b^{2}(z) U_{c l}^{2}(z)\right) & =\frac{2 F_{0}}{\lambda^{2} K U_{c l}(z)}+\frac{2 F_{0}}{\left(2+\lambda^{2}\right) g K} \frac{d U_{c l}}{d z}
\end{aligned}
$$

Using the following non-dimensional transformations (Crapper \& Baines, 1977), to eliminate $F_{0}$ from Eqs. (55) and (56),

$$
\hat{b}=b\left(\frac{g^{3}}{F_{0}^{2}}\right)^{1 / 5}, \hat{u}=\frac{U_{c l}}{\left(g F_{0}\right)^{1 / 5}}, \hat{z}=z\left(\frac{g^{3}}{F_{0}^{2}}\right)^{1 / 5}, N=\hat{b}^{2} \hat{u}, M=\hat{b}^{2} \hat{u}^{2}
$$


where $\hat{b}, \hat{u}, \hat{z}$ are known as top-hat variable (Thomas, 1963; Rooney \& Linden, 1996) and are defined respectively as dimensionless radius, centerline velocity and vertical coordinate of the plume. $N$ and $M$ are the dimensionless mass and momentum respectively. Using the transformations (63), Eqs. (61) and (62) can be given in the form,

$$
\begin{gathered}
\frac{d N}{d \hat{z}}=2 \alpha M^{1 / 2}\left(1-\frac{1}{K N}\right)^{1 / 2} \\
\frac{d M}{d \hat{z}}=\left(\frac{1}{K N-A}\right)\left(\frac{2 N}{\lambda^{2} M}-2 \alpha A \frac{M^{3 / 2}}{N}\left(1-\frac{1}{K N}\right)^{1 / 2}\right)
\end{gathered}
$$

where $A=2 /\left(2+\lambda^{2}\right)$.

The corresponding initial conditions are,

$$
N_{0}=\frac{1}{\left(K\left(\rho_{\infty}-\rho_{0}\right) / \rho_{\infty}\right)}, \quad M_{0}=\frac{\left(U_{0}^{2} / g b_{0}\right)^{2 / 5}}{\left(K\left(\rho_{\infty}-\rho_{0}\right) / \rho_{\infty}\right)^{6 / 5}}
$$

It is common in literature to use the dimensionless height $z / d$ to express the jet traveling distance from the source; so it will be more convenience to do the same in the current study. Using formulas (63), we can obtain the following relation between $\hat{z}$ and $z$,

$$
\frac{z}{d}=\frac{\hat{z}}{\hat{b}_{0}}=\frac{\hat{z} \sqrt{M_{0}}}{N_{0}}
$$

Also, the dimensionless centerline velocity and the jet/plume width can be related to their top-hat variables by the following relations,

$$
\begin{aligned}
& \frac{b}{d}=\frac{\hat{b}}{\hat{b}_{0}}=\frac{N}{N_{0}} \sqrt{\frac{M_{0}}{M}} \\
& \frac{U_{c l}}{U_{0}}=\frac{\hat{u}}{\hat{u}_{0}}=\frac{M}{M_{0}} \frac{N_{0}}{N}
\end{aligned}
$$

\subsection{Centerline quantities}

In order to determine of the centerline quantities, the above non-linear first order ordinary differential system (64)- (65) with the associated initial conditions (66) are integrated numerically. With the aid of the above definition of the buoyancy flux $F$ as well as the relation between density and mass fraction the centerline velocity and jet width and therefore centerline density and centerline concentration can be determined. The solutions are obtained for hydrogen-air buoyant jet, for various values of Froude number $F r=99\left(U_{0}=49.7\right)$, $F r=152(F r=76)$ and $F r=268\left(U_{0}=134\right)$; as used for experiments by (Houf \& Schefer, 2008; Schefer et al., 2008a), at $d=0.00191, \lambda=0.83$, and $\Delta \rho_{0} / \rho_{\infty}=0.93$. The entrainment coefficient $\alpha$ is considered as a variable given by Eq. (41). The centerline hydrogen mass fraction $\mathrm{C}_{c l}$ for $\mathrm{H}_{2}$-air jet at $\mathrm{Fr}=99, \mathrm{Fr}=152$ and $\mathrm{Fr}=268$ are plotted in Fig.7 and compared with the theoretical asymptotic limits for momentum-dominated jets $\left(1 / C_{c l} \propto z\right)$ and the buoyancy-dominated plume limit $\left(1 / C_{c l} \propto z^{5 / 3}\right)$. 


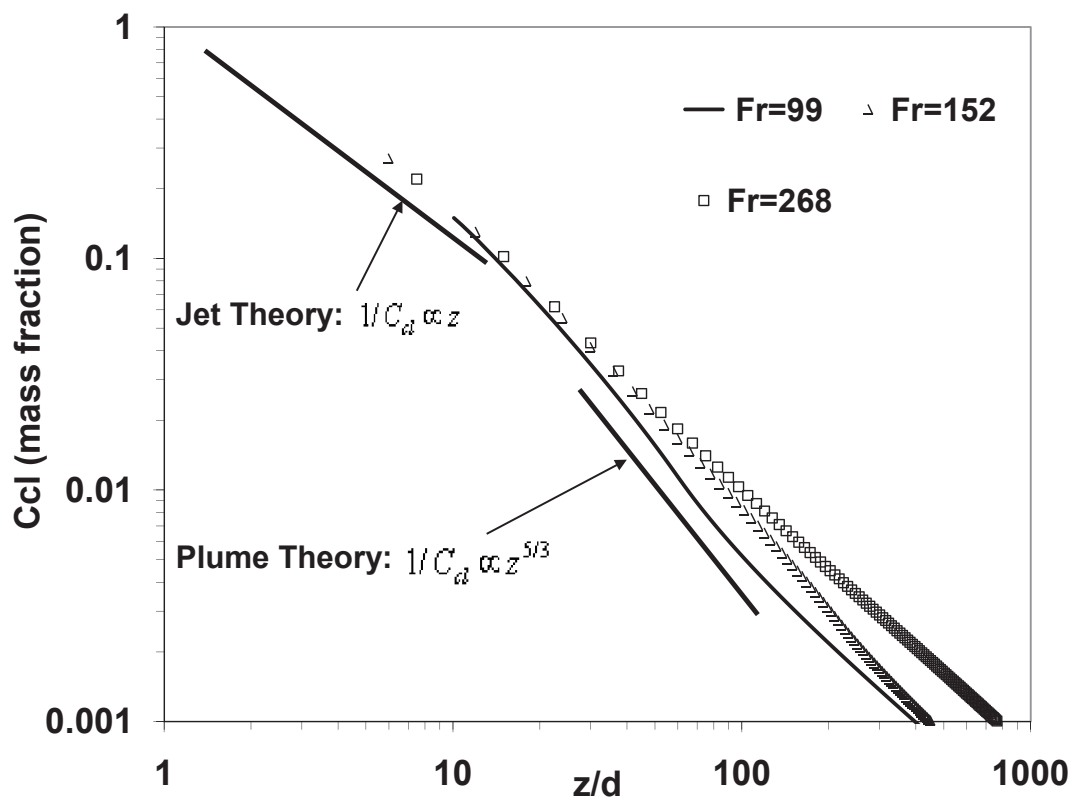

Fig. 7. Comparison of the current model and jet theory of the centerline mass fraction (log-log plot) for $\mathrm{H}_{2}-$ Air jet, with different values of Froude number.

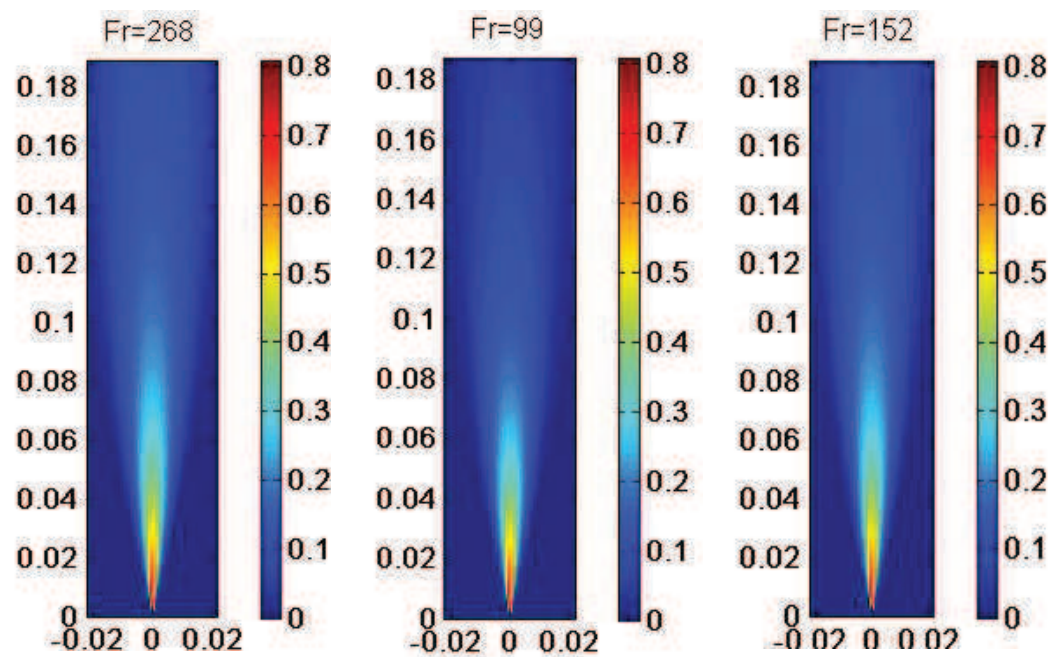

Fig. 8. Contours of the mean mole fraction for $\mathrm{H}_{2}-\mathrm{Air}$ jet with different values of Froude number, with different values of Froude number. 


\subsection{Mean quantities}

Using the mean centerline quantities obtained above with the Gaussian profiles assumptions of axial velocity, density deficiency and mass fraction, Eqs. (31)- (33), we can create a map of the jet concentration and velocity. Using the relation between the mass fraction, mole fraction and density, contours of mole fraction concentration are plotted in Fig.8. Fig. 9 shows a comparison between the present calculations and experiments results (Schefer et al., $2008 \mathrm{~b}$ ) for radial profiles of the mean mass fraction concentration at several axial locations for momentum-dominated $(\mathrm{Fr}=268) \mathrm{H}_{2}-$ Air jet. The maximum centerline mass fraction at each axial location occurs and decreases outward toward the surrounding air. Also, the same figure indicates that the centerline concentration decreases in the downstream direction due to mixing with the ambient air.

Variations of the normalized mean density across the He-Air jet for axial locations from $z / d=$ 50 to 120 and compared with the experimental results by (Panchapakesan \& Lu, 1993) at $d=$ $0.00612, \lambda=0.84$, and $\Delta \rho_{0} / \rho_{\infty}=0.86$ are shown in Fig.10. The maximum deviation of the present model from the experimental results in (Panchapakesan \& Lu, 1993) is around $4 \%$ for the normalized density. It is worth mentioning that for $\mathrm{He}-\mathrm{Air}$ jet the entrainment coefficient is taken as a constant while it is considered as a variable as explained above for calculations of $\mathrm{H}_{2}-\mathrm{Air}$ jet. The mean cross-stream velocity can be obtained by solving the continuity equation for the time-averaged velocities by substituting from (33) and (32) into (28). Fig.12 shows the normalized cross-stream velocity profiles $V / U_{c l}$ plotted against the normalized coordinate $r / c z$ for $\mathrm{H}_{2}-$ Air jet with various values of Froude number $\mathrm{Fr}=268,152$ and 99 . It can be seen from this figure that the cross-stream velocity vanishes at the centerline and becomes outward in the neighborhood of the centerline. A maximum is then reached, then declines back to zero and then becomes inward $\left(V / U_{c l}<0\right)$, reaches a minimum and reaches an asymptote of zero as $r / c z \rightarrow \infty$. The cross-stream velocity asymptotes to zero much more slowly than the streamwise velocity does, thus, the central region is dominated by the axial component of velocity, while the cross-stream flow predominates far away from it. Also,

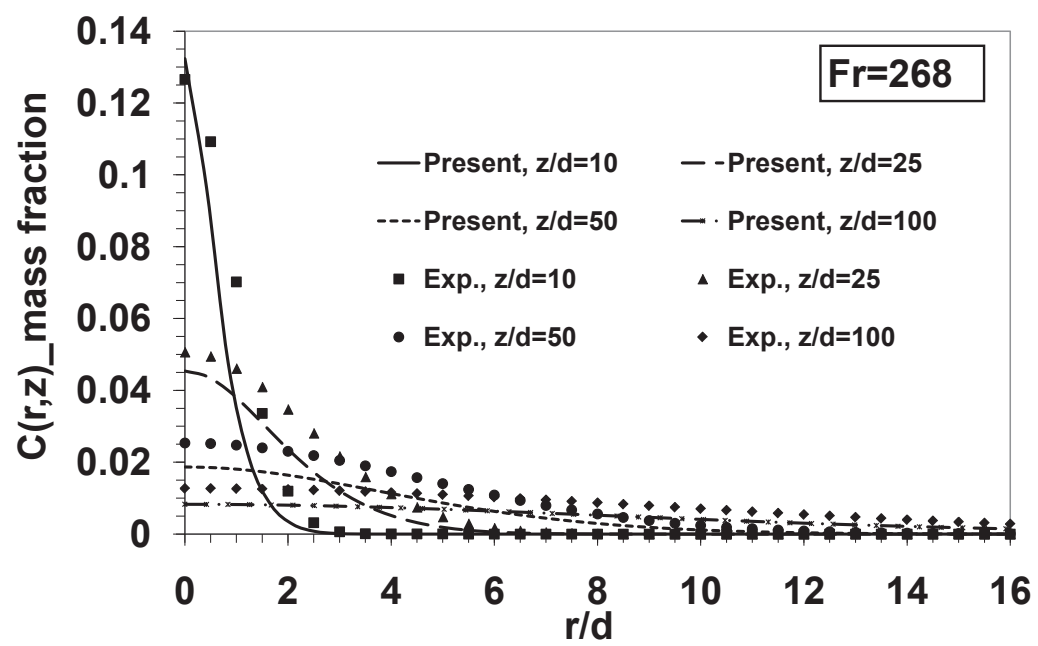

Fig. 9. Radial profiles of the mean mass fraction for momentum-dominated ( $\mathrm{Fr}=268) \mathrm{H}_{2}-$ Air jet at several axial locations, compared with experimental results (Schefer et al., 2008b). 


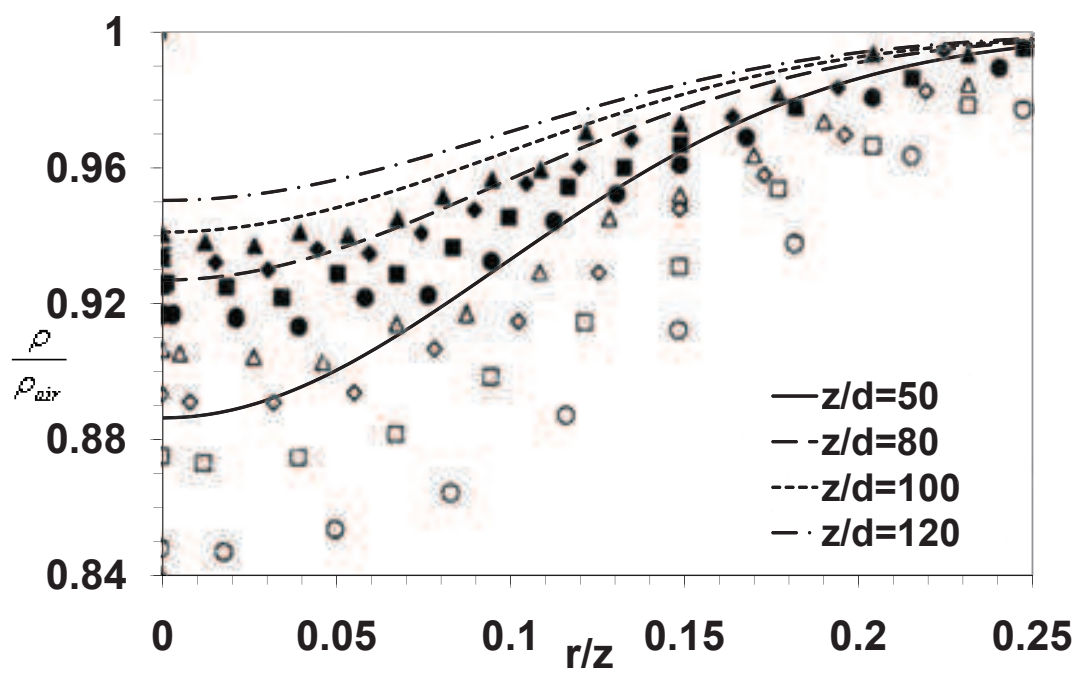

Fig. 10. Radial profiles of the normalized mean density deficiency for $\mathrm{He}-\mathrm{Air}$ jet at several axial locations. Dots refer to experimental results (Panchapakesan \& Lu, 1993) at axial locations from $\mathrm{z} / \mathrm{d}=50$ to 120 .

Fig.11 indicates that as Froude number decreases and the distance from source increases the spreading rate increases and therefore the outward velocity increases.

\subsection{Selected turbulent quantities}

In this section, we determine some of the most important turbulent quantities such as Reynolds stress, velocity-concentration correlation, etc. By substituting the time-averaged

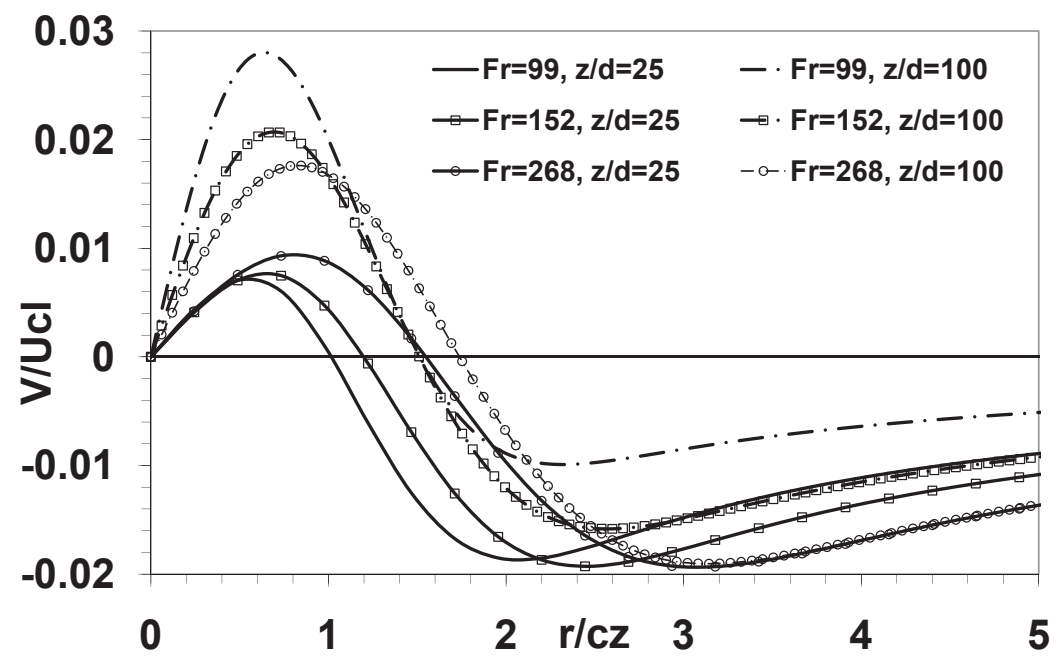

Fig. 11. Radial profiles of the mean cross-stream velocity of $H_{2}-$ Air jet with different values of Froude number at several axial locations. 
profiles of $U, \rho$ and $V$ obtained above into the momentum equation (29), we obtain the time-averaged profile for the Reynolds stress $\overline{u v}$. The radial profiles of normalized Reynolds stress are plotted against the normalized coordinate $\mathrm{r} / \mathrm{cz}$ at several axial locations for $\mathrm{H}_{2}-\mathrm{Air}$ jet with various values of Froude number $F r=268,152$ and 99, as shown in Fig.12. It is observed from this figure that the Reynolds stress $\overline{u v} / U_{c l}^{2}$ increases as the Froude number decreases due to increasing of jet spreading rate.

Again, the time-averaged profile for the velocity-concentration correlation (radial flux) $\overline{v c}$ is determined by inserting the time-averaged profiles of $U, \rho$ and $C$ into the concentration equation (30). The radial profiles of normalized velocity-concentration correlation are plotted in Fig.13 against the normalized coordinate $r / c z$ at several axial locations for $\mathrm{H}_{2}-$ Air jet with various values of Froude number $F r=268,152$ and 99. This figure indicates that the velocity-concentration correlation increases as the Froude number decreases. The turbulent eddy viscosity $v_{t}$ is related to Reynolds stress and the mean axial velocity by the relation,

$$
\overline{u v}=-v_{t} \frac{\partial U}{\partial r}
$$

while, the turbulent eddy diffusivity $D_{t}$ are related to the radial flux and the mean concentration by the relation,

$$
\overline{v c}=-D_{t} \frac{\partial C}{\partial r}
$$

Using current calculation we can determine the turbulent eddy viscosity $v_{t}$ and turbulent eddy diffusivity $D_{t}$. Also, both the normalized turbulent eddy viscosity $v_{t} / U_{c l} b$, and the normalized turbulent eddy diffusivity $D_{t} / U_{c l} b$, are plotted in Fig.14 against the normalized coordinate $\mathrm{r} / \mathrm{cz}$ at several axial locations for momentum-dominated $\mathrm{H}_{2}-\mathrm{Air}$ jet $(\mathrm{Fr}=268)$. Fig.14 illustrates that both $v_{t} / U_{c l} b$ and $D_{t} / U_{c l} b$ have maximum values at the centerline of the jet and decay to zero for large $r$. If the effects of molecular transport are neglected, the turbulent Schmidt number in the free turbulent flow may be defined as the quantitative difference between the turbulent transport rate of material and momentum. In other words, the turbulent $S c h m i d t$ number, $S c_{t}=v_{t} D_{t}$, is a ratio of the eddy viscosity of the turbulent flow

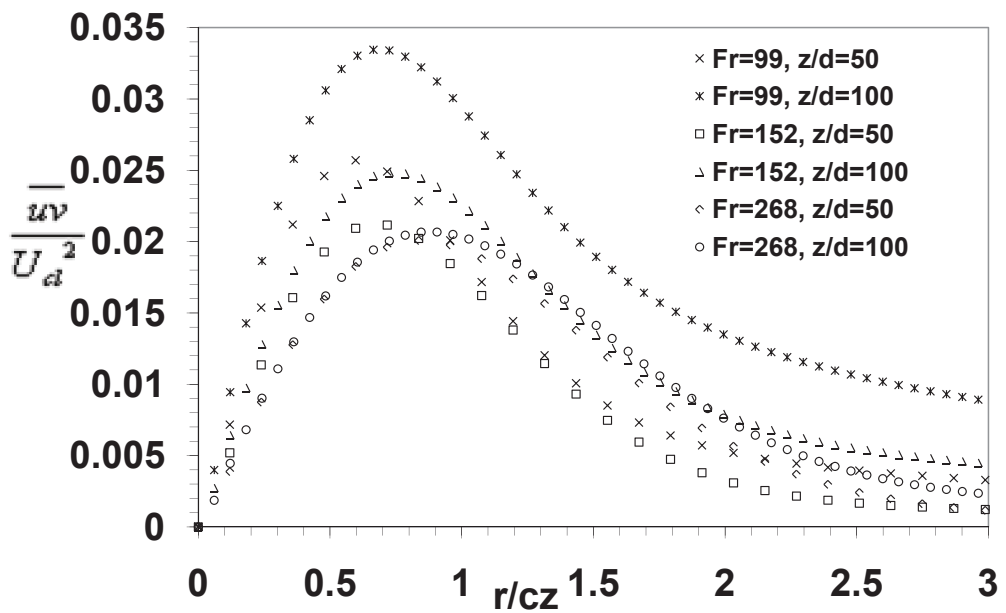

Fig. 12. Radial profiles of the Reynolds stress for $\mathrm{H}_{2}-\mathrm{Air}$ jet with different values of Froude number at several axial locations. 


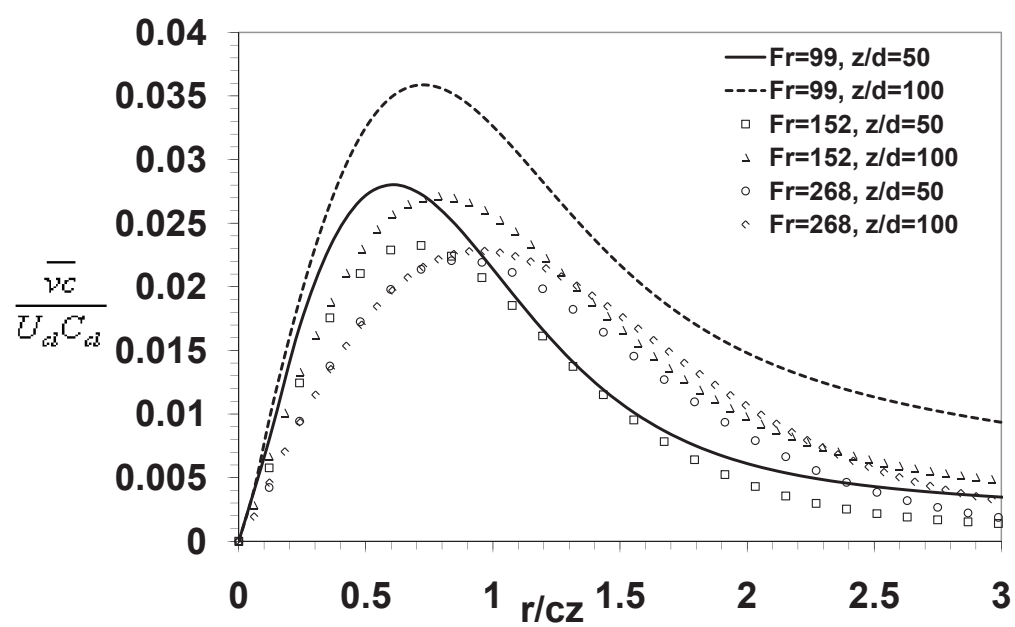

Fig. 13. Radial profiles of the velocity-concentration correlation for $\mathrm{H}_{2}-$ Air jet with different values of Froude number at several axial locations.

$v_{t}$ to the eddy material diffusivity $D_{t}$. It is very well known from literature that the spreading rate of material in free jets is greater than that of momentum; thus, the turbulent Schmidt number is less than unity and has a significant effect on the predictions of species spreading rate in jet flows. The turbulent eddy viscosity $v_{t}$ and the turbulent eddy diffusivity $D_{t}$, give the turbulent Schmidt number as,

$$
S c_{t}=\frac{v_{t}}{D_{t}}
$$

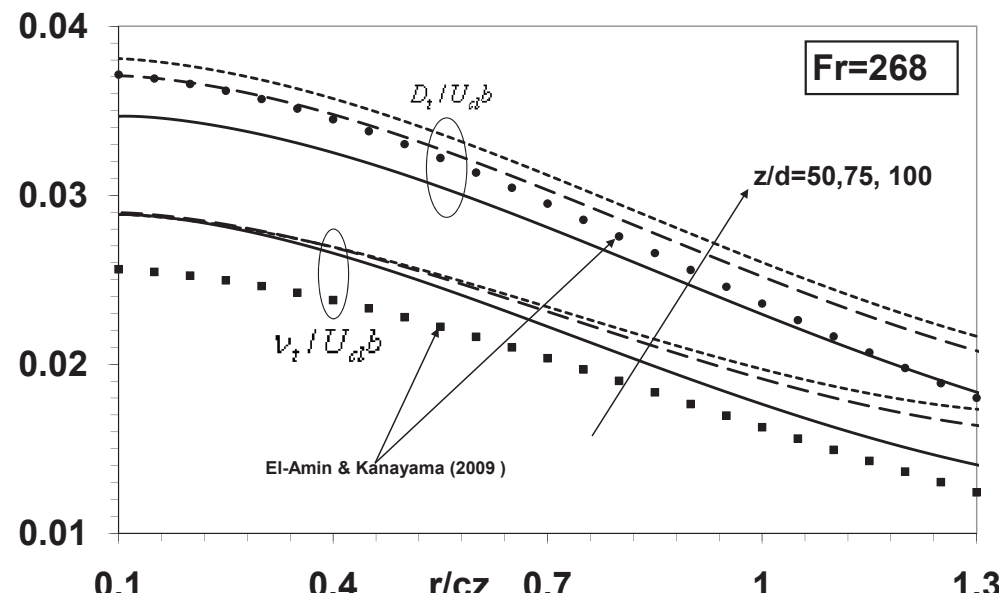

Fig. 14. Radial profiles of turbulent eddy viscosity and turbulent eddy diffusivity for $\mathrm{H}_{2}-\mathrm{Air}$ with $\mathrm{Fr}=268$ (momentum-dominated) at several axial locations, compared with (El-Amin, 2009). 


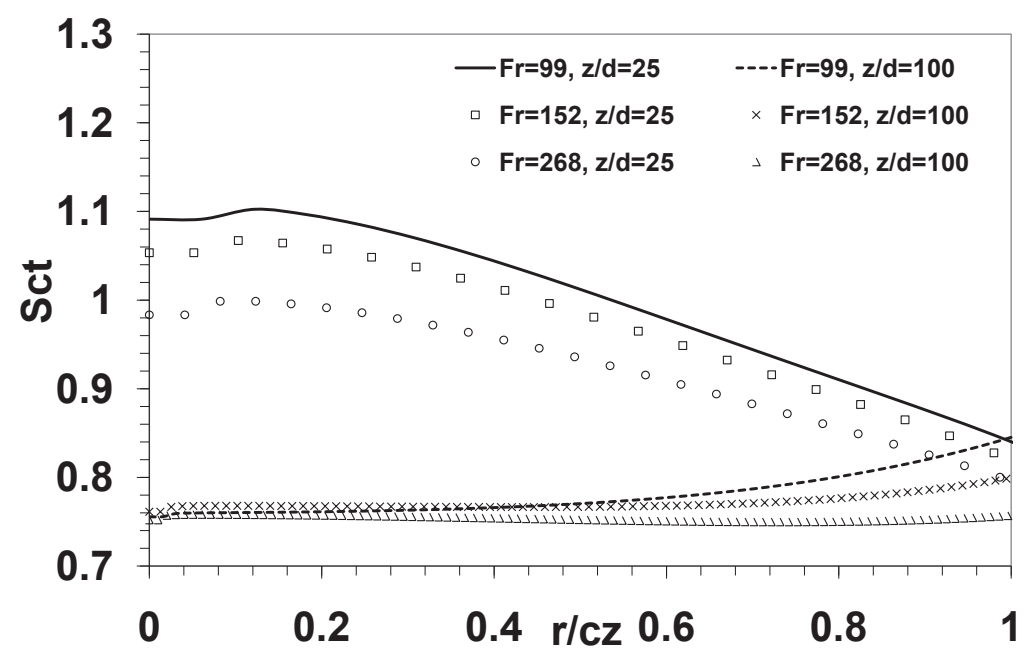

Fig. 15. Radial profiles of turbulent Schmidt number for $\mathrm{H}_{2}-$ Air jet with different values of Froude number at several axial locations.

Turbulent Schmidt number is plotted in Fig.15 against the normalized coordinate $r / c z$ at several axial locations for $\mathrm{H}_{2}-\mathrm{A}$ ir jet with various values of Froude number $\mathrm{Fr}=268,152$ and 99. In the momentum-dominated region the turbulent Schmidt number seems to be constant and the opposite is true when the buoyancy effects take place for small Froude number or far distance from the jet source. Irrespective of the turbulence structure, the constant value of the turbulent Schmidt number across the whole flow field implies that the momentum process is similar to the material-transport process. The opposite is true for the variable values of the turbulent Schmidt number indicate that the momentum process is not similar to the material-transport process. Now, let us define the normalized streamwise momentum flux density across an isothermal jet as, $G=\left(U / U_{c l}\right)^{2}$, and the normalized jet-feed material density as, $W=U C / U_{c l} C_{c l}$. Also, by considering the analytical correlation of jet-feed concentration and streamwise velocity derived by (El-Amin \& Kanayama, 2009b) as, $W=G\left(1+S c_{t}\right) / 2$ (see Fig.6).

\section{References}

Agrawal, A. \& Prasad, A. K. (2002). Properties of vortices in the self-similar turbulent jet, Exp. Fluids 33: 565-577.

Agrawal, A. \& Prasad, A. K. (2003). Integral solution for the mean flow profiles of turbulent jets, plumes, and wakes, ASME J Fluids Engineering 125: 813-822.

Batchelor, G. K. (1954). Heat convection and buoyancy effects in fluids, Q. J. R. Met. Soc. 80: 339-358.

Becker, H. A., Hottel, H. C. \& Williams, G. C. (1967). The nozzle-fluid concentration of the round turbulent free jet, J. Fluid Mech 30: 285-303.

Bhat, G. S. \& Krothapalli, A. (2000). Simulation of a round jet and a plume in a regional atmospheric model, Monthly Weather Review 128: 4108-4117.

Bhat, G. S. \& Narasimha, R. A. (1996). A volumetrically heated jet: Large-eddy structure and 
entrainment characteristics, J. Fluid Mechanics 325: 303-330.

Carlotti, P. \& Hunt, G. R. (2005). Analytical solutions for turbulent non-boussinesq plumes, J. Fluid Mechanics 538: 343-359.

Chen, C. J. \& Rodi, W. (1980). Vertical turbulent buoyant jets - a review of experimental data, Pergamon Press, Oxford, UK.

Crapper, P. F. \& Baines, W. D. (1977). Non-boussinesq forced plumes, Atmospheric Environment 11: 415-420.

Dai, Z., Tseng, L. K. \& Faeth, G. M. (1995). Velocity statistics of round, fully developed, buoyant turbulent plumes, Trans. ASME, J. Heat Transfer 117: 138-145.

El-Amin, M. F. (2009). Non-boussinesq turbulent buoyant jet resulting from hydrogen leakage in air, Int. J. Hydrogen Energy 34: 7873-7882.

El-Amin, M. F., Inoue, M. \& H., K. (2008). Boundary layer theory approach to the concentration layer adjacent to a ceiling wall of a hydrogen leakage: far region, Int. J. Hydrogen Energy 33: 7642-7647.

El-Amin, M. F. \& Kanayama, H. (2008). Boundary layer theory approach to the concentration layer adjacent to a ceiling wall at impinging region of a hydrogen leakage, Int. J. Hydrogen Energy 33: 6393-6400.

El-Amin, M. F. \& Kanayama, H. (2009a). Boundary layer theory approach to the concentration layer adjacent to the ceiling wall of a hydrogen leakage: axisymmetric impinging and far regions, Int. J. Hydrogen Energy 34: 1620-1626.

El-Amin, M. F. \& Kanayama, H. (2009b). Integral solutions for selected turbulent quantities of small-scale hydrogen leakage: a non-buoyant jet or momentum-dominated buoyant jet regime, Int. J. Hydrogen Energy 34: 1607-1612.

El-Amin, M. F. \& Kanayama, H. (2009c). Similarity consideration of the buoyant jet resulting from hydrogen leakage, Int. J. Hydrogen Energy 34: 5803-5809.

El-Amin, M. F., Sun, S., Heidemann, W. \& Mller-Steinhagen, H. (2010). Analysis of a turbulent buoyant confined jet using realizable k-jepi model, Heat and Mass Transfer To appear: 1-20.

Fischer, H. B., List, E. J., Koh, R. C. Y., Imberger, J. \& Brooks, N. H. (1979). Mixing in Inland and Coastal Waters, Academic Press.

Gebhart, B., Jaluria, Y., Mahajan, R. L. \& Sammakia, B. (1988). Buoyancy-induced flows and transport, Hemisphere. Washington, DC.

He, G., Yanhu, G. \& A., H. (1999). The effect of schmidt number on turbulent scalar mixing in a jet-in-cross flow, Int. J. Heat Mass Transfer 42: 3727-3738.

Hinze, J. O. (1975). Turbulence, 2nd ed., McGraw-Hill.

Hirst, E. A. (1971). Analysis of buoyant jets within the zone of flow establishment, Technical report, Oak Ridge National Laboratory, Report ORNL-TM-3470.

Houf, W. G. \& Schefer, R. W. (2007). Predicting radiative heat fluxes and flammability envelopes from unintended releases of hydrogen, Int. J. Hydrogen Energy 32: 136-151.

Houf, W. G. \& Schefer, R. W. (2008). Analytical and experimental investigation of small-scale unintended releases of hydrogen, Int. J. Hydrogen Energy 33: 1435-1444.

Hussein, J. H., Capp, S. P. \& George, W. K. (1994). Velocity measurements in a high-reynolds-number, momentum-conserving, axisymmetric, turbulent jet, J. Fluid Mechanics 258: 31-75.

Lessen, M. (1978). On the power laws for turbulent jets, wakes and shearing layers and their relationship to the principal of marginal instability, J. Fluid Mech 88: 535-540.

List, E. J. (1982). Turbulent jets and plumes, Annual Review of Fluid Mechanics 14: 189-212. 
Morton, B. R. (1965). Modelling fire plumes, 10th Int. Symposium on Combustion, 844-859.

Panchapakesan, N. R. \& Lu (1993). Turbulence measurements in axisymmetric jets of air and helium. part 2. helium jet, J. Fluid Mechanics 246: 225-247.

Panchapakesan, N. R. \& Lumley, J. L. (1993). Turbulence measurements in axisymmetric jets of air and helium. part 1. air jet, J. Fluid Mechanics 246: 197-223.

Papantoniou, D. \& List, E. J. (1989). Large-scale structure in the far field of buoyant jets, J. Fluid Mechanics 209: 151-190.

Rajaratnam, N. (1976). Turbulent Jets, Elsevier Science, Place of publication.

Ricou, F. \& Spalding, D. B. (1961). Measurements of entrainment by axisymmetrical turbulent jets, J. Fluid Mechanics 8: 21-32.

Rooney, G. G. \& Linden, P. F. (1996). Similarity considerations for non-boussinesq plumes in an unstratified environment, J. Fluid Mechanics 318: 237-250.

Schefer, R. W., Houf, W. G., Bourne, B. \& Colton, J. (2006). Spatial and radiative properties of an open-flame hydrogen plume, Int. J. Hydrogen Energy 31: 1332-1340.

Schefer, R. W., Houf, W. G. \& Williams, T. C. (2008a). Investigation of small-scale unintended releases of hydrogen: buoyancy effects, Int. J. Hydrogen Energy 33: 4702-4712.

Schefer, R. W., Houf, W. G. \& Williams, T. C. (2008b). Investigation of small-scale unintended releases of hydrogen: momentum-dominated regime, Int. J. Hydrogen Energy 33: 6373-6384.

Shabbir, A. \& Georg, W. K. (1994). Experiment on a round turbulent plume, J. Fluid Mechanics 275: $1-32$.

Spiegel, E. A. \& Veronis, G. (1960). On the boussinesq approximation for a compressible fluid, Astrophys. J. 131: 442-447.

Steward, F. R. (1970). Prediction of the height of turbulent diffusion buoyant flames, Combust. Sci. Technol. 2: 203-212.

Swain, M. R., Filoso, P. A. \& Swain, M. N. (2007). An experimental investigation into the ignition of leaking hydrogen, Int. J. Hydrogen Energy 32: 287-295.

Takeno, K., Okabayashi, K., Kouchi, A., Nonaka, T., Hashiguchi, K. \& Chitose, K. (2007). Dispersion and explosion field tests for 40 mpa pressurized hydrogen, Int. J. Hydrogen Energy 32: 2144-2153.

Tennekes, H. \& Lumley, J. L. (1972). A First Course in Turbulence, MIT Press, Cambridge, MA.

Thomas, P. H. (1963). The size of flames from natural fires, 9th Symposium on Combustion, 844-859.

Townsend, A. A. (1966). The mechanism of entrainment in free turbulent flows, J. Fluid Mechanics 26: 689-715.

Turner, J. S. (1973). Buoyancy effects in fluids, Cambridge Univ. Press, London.

Turner, J. S. (1986). Turbulent entrainment: The development of the entrainment assumption, and its application to geophysical flows, J. Fluid Mech 173: 431-471.

Venkatakrishnan, L., Bhat, G. S. \& Narasimha, R. (1999). Experiments on plume with off-source heating: Implications for cloud fluid dynamics, J. Geophys. Res. 104: 14271-14281.

Woods, A. W. (1997). A note on non-boussinesq plumes in an incompressible stratified environment, J. Fluid Mechanics 345: 347-356.

Yimer, I., Campbell, I. \& Jiang, L. Y. (2002). Estimation of the turbulent schmidt number from experimental profiles of axial velocity and concentration for high-reynolds-number jet flows, Canadian Aeronautics Space Journal 48: 195-200. 


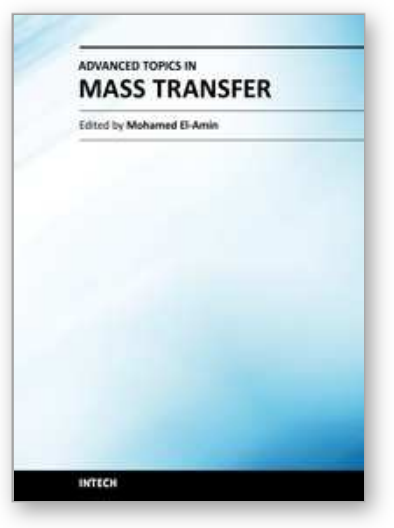

\author{
Advanced Topics in Mass Transfer \\ Edited by Prof. Mohamed El-Amin
}

ISBN 978-953-307-333-0

Hard cover, 626 pages

Publisher InTech

Published online 21, February, 2011

Published in print edition February, 2011

This book introduces a number of selected advanced topics in mass transfer phenomenon and covers its theoretical, numerical, modeling and experimental aspects. The 26 chapters of this book are divided into five parts. The first is devoted to the study of some problems of mass transfer in microchannels, turbulence, waves and plasma, while chapters regarding mass transfer with hydro-, magnetohydro- and electro- dynamics are collected in the second part. The third part deals with mass transfer in food, such as rice, cheese, fruits and vegetables, and the fourth focuses on mass transfer in some large-scale applications such as geomorphologic studies. The last part introduces several issues of combined heat and mass transfer phenomena. The book can be considered as a rich reference for researchers and engineers working in the field of mass transfer and its related topics.

\title{
How to reference
}

In order to correctly reference this scholarly work, feel free to copy and paste the following:

M.F. El-Amin and Shuyu Sun (2011). Turbulent Buoyant Jet of a Low-Density Gas Leaks into High-Density Ambient: Hydrogen Leakage in Air, Advanced Topics in Mass Transfer, Prof. Mohamed El-Amin (Ed.), ISBN: 978-953-307-333-0, InTech, Available from: http://www.intechopen.com/books/advanced-topics-in-masstransfer/turbulent-buoyant-jet-of-a-low-density-gas-leaks-into-high-density-ambient-hydrogen-leakage-in-air

\section{INTECH}

open science | open minds

\author{
InTech Europe \\ University Campus STeP Ri \\ Slavka Krautzeka 83/A \\ 51000 Rijeka, Croatia \\ Phone: +385 (51) 770447 \\ Fax: +385 (51) 686166 \\ www.intechopen.com
}

\author{
InTech China \\ Unit 405, Office Block, Hotel Equatorial Shanghai \\ No.65, Yan An Road (West), Shanghai, 200040, China \\ 中国上海市延安西路65号上海国际贵都大饭店办公楼 405 单元 \\ Phone: +86-21-62489820 \\ Fax: +86-21-62489821
}


(C) 2011 The Author(s). Licensee IntechOpen. This chapter is distributed under the terms of the Creative Commons Attribution-NonCommercialShareAlike-3.0 License, which permits use, distribution and reproduction for non-commercial purposes, provided the original is properly cited and derivative works building on this content are distributed under the same license. 